\title{
Philosophiques
}

\section{Féminisme et postcolonial : quel projet pour la critique ?}

\section{JULIE SAADA}

Volume 44, numéro 1, printemps 2017

URI : https://id.erudit.org/iderudit/1040335ar

DOI : https://doi.org/10.7202/1040335ar

Aller au sommaire du numéro

Éditeur(s)

Société de philosophie du Québec

ISSN

0316-2923 (imprimé)

1492-1391 (numérique)

Découvrir la revue

Citer ce document

Saada, J. (2017). Féminisme et postcolonial : quel projet pour la critique ?

Philosophiques, 44(1), 139-143. https://doi.org/10.7202/1040335ar d'utilisation que vous pouvez consulter en ligne.

https://apropos.erudit.org/fr/usagers/politique-dutilisation/ 


\title{
Féminisme et postcolonial : quel projet pour la critique?
}

\author{
JULIE SAADA \\ Sciences Po, Ecole de droit, Paris, France' \\ julie.saada@sciencespo.fr
}

Les théories postcoloniales et le féminisme constituent des domaines entiers de la philosophie contemporaine. Ils sont aussi l'objet de controverses, scissions, déconstructions et reconstructions qui marquent un renouvellement de la théorie critique. Avec Décoloniser le féminisme, Soumaya Mestiri offre une perspective théorique et conceptuelle dont la particularité est de confronter les catégories philosophiques aux contextes de domination, de les mettre à l'épreuve de situations singulières et d'inclure les discours d'acteurs dont les expériences situées sont prises au sérieux, tout en les inscrivant dans un projet normatif. Car la perspective critique forte est salutairement articulée à un projet reconstructif qui entend renouveler le lien entre approche empirique et perspective normative en philosophie.

L'un des apports précieux de l'ouvrage réside dans les références mobilisées. Soumaya Mestiri fait entrer dans le débat philosophique - on peut saluer au passage le choix éditorial engagé - des auteurs relativement peu connus du féminisme, de la théorie critique, de l'histoire littéraire ou herméneutique de l'Islam, ou encore des débats tunisiens de l'après-Révolution. De tels apports mériteraient de faire l'objet d'une nouvelle publication, sous la forme d'une anthologie ou d'une présentation systématique, non seulement parce qu'ils permettraient de mieux saisir les perspectives philosophiques riches, pointues, exigeantes, que développe Soumaya Mestiri, mais aussi parce qu'en eux-mêmes ils favoriseraient et renforceraient la pluralité des sources intellectuelles au sein de la philosophie critique.

Le titre de l'ouvrage indique que ce dernier s'inscrit dans une perspective normative déterminée: «décoloniser» le féminisme requiert un geste critique au sein de la critique elle-même, qui montre la manière dont la critique (féministe) peut véhiculer des concepts, des postures théoriques et produire des effets anti-critiques dès lors qu'elle s'inscrit dans un programme occidentalo-centré qui reproduit les dominations coloniales au-delà des décolonisations des années 1960 et assure le maintien de hiérarchies structurelles, notamment portées par un concept d'universel. Parce que le féminisme majoritaire conteste les dominations de genre tout en prétendant dépasser les dominations sous la forme d'une émancipation pensée pour toutes et tous, il n'émanciperait en réalité que certaines femmes et ce, au prix de la subordination des autres. Somme toute, et pour résumer de façon

1. Sciences Po est membre de l'USPC.

PHILOSOPHIQUES 44/1 — Printemps 2017, p. 139-143 
réductrice - eu égard à la complexité et à la finesse des arguments déployés par l'auteure - les femmes occidentales ont gagné leur émancipation au prix de la subordination des femmes indigènes. L'universel est ainsi le nom d'une pensée blanche, laïque, d'une vision impériale de la femme dont le corollaire est l'orientalisation des autres femmes, que conteste et déconstruit Soumaya Mestiri dans un double horizon politique et épistémologique.

La déconstruction menée par l'auteure porte autant sur le féminisme «majoritaire» qui occulterait son propre échec que sur la pensée libérale incarnée par Rawls, sur les théories de l'empowerment et leurs usages dans le tiers-monde, ou sur le féminisme musulman qui aurait failli à proposer une solution décoloniale au féminisme laïc alors même que l'une des sources majeures auxquelles on attribue la subordination des femmes musulmanes, le Coran, pourrait être interprétée dans un sens égalitaire, distinct des discours essentialistes de la complémentarité. Toute une tradition de littérature érotique en Islam, soutient l'auteure, affirme d'ailleurs l'égalité sexuelle et permettrait de balayer les clichés sur l'islam réduit au statut qu'il confère aux femmes. Féminismes alternatifs (Sarah Song, Fatima Mernissi) et libéraux sont renvoyés dos à dos dès lors qu'ils échouent à penser la différence tout en reproduisant les structures hégémoniques.

Sur le plan normatif, Soumaya Mestiri défend un «différentialisme bien compris » (p. 84), modalité de l'égalité et lié à la solidarité, qui porte les voix des récits subalternes ou "indigènes" dans le cadre d'une "épistémé pluritopique ", et s'inscrit dans une approche transculturelle, polycentrée, pensée dans une perspective non consensuelle donc non libérale. L'auteure entend développer un «féminisme de la frontière» qui récuse les exclusions essentielles et procède en dé-margeant les savoirs pour les faire dialoguer à égalité. L'ouvrage de Soumaya Mestiri œuvre en ce sens tant, répétons-le, la mobilisation d'auteurs non mainstream est l'un des apports majeurs de la critique comme du projet normatif qui est formulé.

Un certain nombre de points s'offrent cependant à la discussion.

I) S'il ne va pas de soi qu'il existe un féminisme majoritaire ayant pour caractéristique première d'être blanc, occidental donc dominateur, tant les féminismes sont variés, c'est l'usage des catégories générales fait par l'auteure qui peut déconcerter le lecteur. Soumaya Mestiri souligne en effet, d'un côté, que le "féminisme de la frontière" se constitue précisément par les intersections, le transculturel, le polycentrisme, qui sont autant de caractéristiques signalant l'instabilité et la porosité des catégories comme des identités, de l'autre, que l'essentialisme stratégique offre dans le féminisme comme dans les études postcoloniales un outil pertinent. Mais l'usage par l'auteure de catégories essentialisées réalise-t-il son objectif stratégique? Comment comprendre l'usage permanent et exclusif de catégories construites en oppositions simples — les femmes «indigènes", «subalternes », «colonisées", "musulmanes», "simples croyantes», "marginales» contre les femmes «blanches", «laïques", « occidentales », « universalistes» — sinon comme 
l'assignation simplifiée à des identités fixes, essentialisées et hermétiquement closes? Pourquoi les identités de genre peuvent-elles être déconstruites, mais pas les identités postcoloniales?

On peut se demander, par exemple, comment sont pensées les femmes musulmanes occidentales et, de manière générale, toutes les femmes qui sont précisément à la frontière de ces catégories et signalent l'instabilité de ces dernières. À partir de quand une femme vivant en Occident peut-elle être considérée comme une occidentale? Toute musulmane est-elle une indigène ou existe-t-il à l'intérieur de l'islam des différences? Est-il absolument exclu qu'une «blanche» puisse être «orientale» ou «subalterne»? Être "blanc» ou "subalterne» est-il une essence ou un effet de contexte et de pouvoir? Pour le dire autrement, la stratégie essentialiste ne doit pas empêcher de penser les transformations, détournements, retournements, subversions des identités, y compris "postcoloniales", si l'on veut, dans l'optique de l'auteure, "résister aux essentialisations ethnico-raciales forgées par la colonisation et qui perdurent sous la double colonialité du genre» (p. 97).

De manière plus générale, il me semble que le postcolonial n'est pas la reproduction et la continuation à l'identique de l'histoire des colonies. Il ne présente pas non plus une unité conceptuelle. Enfin, il ne constitue pas le concept explicatif unique des dominations et ne peut pas conduire à saisir toute domination comme postcoloniale. Au contraire, il est sans cesse refaçonné dans des controverses en fonction des stratégies critiques, déconstructives et parfois reconstructives. Une perspective critique cohérente n'implique-t-elle pas, sur le plan épistémologique, de dénoncer la fétichisation des catégories associées au genre et de ne pas fétichiser les catégories associées au postcolonial? Une perspective d'émancipation n'implique-t-elle pas, quant à elle, de penser au-delà des catégories et des savoirs (et de la langue ?) qui produisent ou au moins véhiculent les dominations?

2) L'opposition entre un universel «blanc» et une périphérie «indigène » ou «musulmane» pensée comme le particulier ne rend pas compte, non plus, des "conflits d'universalités " (Balibar). Si la laïcité, évoquée dans l'ouvrage, se présente en effet comme un discours universaliste, il ne va pas de soi que les discours auxquels elle s'oppose (en principe et/ou dans les usages sociaux qui en sont faits) soient relégués dans le particulier, le périphérique et le subalterne. Au contraire, les penser comme des universels concurrents, conflictuels, incompatibles, permet précisément d'éviter l'illusion d'hybridation comme le dépassement dans une posture de surplomb - deux écueils que dénonce par ailleurs l'auteure.

3) Le refus des positions de surplomb, fait par Soumaya Mestiri, constitue un choix épistémologique fructueux tant il met au défi une discipline philosophique historiquement constituée comme pensée du général contre le singulier, ou des catégories abstraites contre les contingences empiriques. Bien qu'il n'aille pas forcément de soi que "vouloir résorber la contradiction à tout prix» soit "une attitude typiquement coloniale» ou 
une manifestation du "syndrome de l'intégration globale» (p. 73) — puisque l'auteure cherche elle-même une cohérence conceptuelle et théorique -, le refus de la pensée surplombante constitue une épistémologie critique. Celleci est déjà largement développée au sein du féminisme comme des usages divers du storytelling - ces récits de domination faits par des agents situés qui subissent les effets de structures sociales, politiques et institutionnelles de domination. On peut cependant se demander si le récit constitue seulement une expression des formes et des vécus de domination ou s'il produit aussi une expérience transformatrice de soi, d'émancipation et, somme toute, d'empowerment. Ce dernier, plus que le care, ne constitue-t-il pas un projet d'émancipation réelle? Plus précisément, entre la domination et l'émancipation, n'y a-t-il pas de place pour une pensée des processus et des transformations, des changements de place et de la porosité des catégories épistémiques et des identités, de sorte que la critique de la pensée surplombante implique de penser le caractère mouvant, instable, de ces catégories et identités?

4) L'un des concepts mobilisés dans la perspective normative déployée par Soumaya Mestiri est celui de dignité. Ce dernier correspond, dans l'ouvrage, au féminisme de la frontière, décolonisé, s'accordant avec un "vivreensemble serein» (p. I50) qui rejette "la version libérale, blanche, du multiculturalisme hégémonique»(p. I 53) pour soutenir la démocratie transculturelle, pluricentrée, radicale (p. I 58 ) donc non libérale, invitant les subalternes à devenir les participants actifs d'une histoire conflictuelle partagée, et auxquels on reconnaitrait «le droit à la dignité ». Le concept de dignité serait la condition de l'égalité qui assume la différence sans redistribuer les hiérarchies (de genre, coloniales), mais en liant par des relations de solidarité. La dignité serait ainsi par excellence le concept et la forme de relation permettant de "décoloniser le genre ».

Cependant, le concept de dignité est controversé, mobilisé dans des projets souvent peu en accord avec l'émancipation et les épistémologies critiques. Qu'il s'agisse (en France, du moins) de libérer - y compris contre leur gré - les femmes musulmanes du voile qui leur serait imposé par les hommes musulmans, de régler le statut du corps humain dans nombre de débats en bioéthique, ou de réduire la liberté de disposer de son propre corps dans des domaines aussi divers que les pratiques sexuelles, la pornographie, la prostitution, la réparation du "préjudice d'être né ", la dignité est un principe normatif de l'État de droit mais aussi un moyen de restreindre les libertés en imposant des obligations plus fondamentales. Les usages de ce concept signalent même un renversement des libertés fondamentales au profit d'obligations naturelles à l'égard de soi-même (O. Cayla, Y. Thomas). En quel sens, et pour quels usages, ce concept peut-il être construit pour être compatible avec un programme d'émancipation des femmes?

5) Si le sens d'un concept dépend en partie des usages qui en sont faits, le sens d'un texte est également relatif à ses interprétations. Soumaya Mestiri 
soutient, contre nombre d'interprétations du Coran, que ce dernier n'est pas anti-féministe mais qu'il est aussi porteur d'un principe d'égalité des sexes. À l'instar des voix et des études féministes remarquablement placées par l'auteure dans le débat philosophique, on ne peut qu'appeler un geste analogue concernant ces interprétations du Coran, tant les caricatures et réductions servent (en France) des programmes politiques aux objectifs largement discriminants. Sur quels types de théorie et de pratique interprétatives Soumaya Mestiri s'appuie-t-elle pour mettre en avant la dimension égalitaire du Texte?

6) Interrogeons au passage l'interprétation de textes érotiques, dont l'auteure déduit, à partir de l'évocation qui y est faite du désir insatiable des femmes et de leur jouissance sexuelle, l'affirmation d'une égalité dans la pratique sexuelle ou d'un "contrat sexuel » qui vient "décoloniser Shéhérazade». Est-il naif d'observer que la supériorité des femmes, lorsqu'elle tient à leur capacité à "épuiser une troupe d'hommes» (p. 53), cantonne cette supériorité au lit (ou bien où l'on voudra) pour mieux maintenir les femmes hors de la cité, et relève d'un fantasme d'homme? Que les textes cités soient d'ailleurs écrits exclusivement par des hommes n'est pas pour surprendre.

7) L'épistémologie définie dans l'ouvrage suscite des attentes: elle porte en elle une approche normative - et pas seulement critique - qui appelle, sinon un programme, du moins des pistes d'action et d'engagement pratique. La critique salutaire de la position de surplomb menée par Soumaya Mestiri appelle des développements théoriques qui prennent les faits au sérieux, ne les relèguent pas dans l'espace sombre et inexploré auquel les confine la théorie philosophique traditionnelle, et ne se contente pas d'opposer les théories entre elles. Comment penser plus avant, et en pratique, la décolonisation du genre, au-delà des solutions présentées par l'auteure, à savoir: le «jeu» et le "voyage» qui permettraient de «troquer l'arrogance contre l'amour» (p. I47) ? Bien qu'il soit associé à la raison libérale (p. I32) ou au colonial (p. I33), mais parce qu'il dépasse «le concept d'autonomie, immanquablement présenté sous une forme machiste et foncièrement patriarcale» (p. I 27), le care suffit-il à remplir cette promesse? L'appendice sur John Stuart Mill, dont on ne saisit pas bien le lien avec le reste de l'ouvrage, n'offre pas de réponse à cette attente ni ne précise le projet normatif pourtant annoncé par le titre même de l'ouvrage.

Ces questions ouvriront, je l'espère, une discussion et - nous pouvons l'espérer au regard de la très grande qualité philosophique de l'ouvrage de nombreux autres travaux à venir de la part de Soumaya Mestiri. 